\title{
Flow Dynamic Analysis by Contrast-Enhanced Imaging Techniques of Medium Cutoff Membrane Hemodialyzer
}

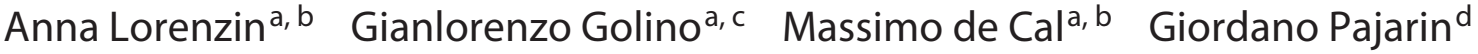 \\ Sergio Savastano $^{d}$ Andrea Lupi $^{\mathrm{e}}$ Alessandra Sandini ${ }^{f}$ Francesco Fiorin $^{f}$ \\ Claudio Ronco ${ }^{\mathrm{a}} \mathrm{b}, \mathrm{g}$
}

\begin{abstract}
anternational Renal Research Institute of Vicenza, Vicenza, Italy; ${ }^{b}$ Department of Nephrology, Dialysis and Transplantation, St. Bortolo Hospital, aULSS8 Berica, Vicenza, Italy; ' Department of Medicine-DIMED, Section of Anesthesiology and Intensive Care Medicine, University of Padova, Padua, Italy; ${ }^{\text {dDepartment }}$ of Radiology,

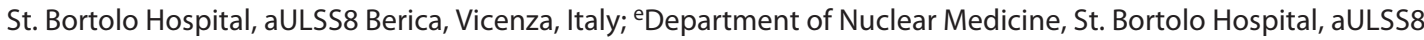
Berica, Vicenza, Italy; 'Department of Transfusion Medicine, St. Bortolo Hospital, aULSS8 Berica, Vicenza, Italy; ${ }^{9}$ Department of Medicine, University of Padua, Padua, Italy
\end{abstract}

\section{Keywords}

Expanded hemodialysis · Flow distribution · Medium cutoff · Internal filtration · Computed tomography imaging

\footnotetext{
Abstract

Introduction: Medium cutoff (MCO) membranes represent an interesting innovation in the field of hemodialysis. Given the correlation between large ( $\mathrm{PM}>25 \mathrm{kDa}$ ) middle molecules (LMM) and clinical outcomes, the possibility to broaden the spectrum of solutes removed in hemodialysis with MCO membranes introduces a new perspective for endstage kidney disease patients. Due to low diffusion coefficients of LMM, the use of convection is required to maximize extracorporeal clearance. High convective rates are achieved with high-flux membranes in hemodiafiltration, a technique not available in the US. In case of the MCO membrane, remarkable clearances of LMM are achieved combining the permeability of the membrane with a significant amount of
}

internal convection. The mechanism of filtration-backfiltration inside the dialyzer enables effective removal of LMM in a technique called expanded hemodialysis (HDx). Given such theoretical explanation, it is important to demonstrate the blood and ultrafiltration rheology inside the MCO dialyzer. Method: This study for the first time describes flow dynamic parameters and internal cross-filtration, thanks to specific radiology and nuclear imaging techniques. Results: Flow dynamic analysis of the blood and dialysate compartment confirms excellent distribution of velocities and an excellent matching of blood and dialysate. Average blood flow velocity allows for wall shear rates adequate to avoid protein stagnation at the blood membrane interface and increase in blood viscosity. Cross-filtration analysis demonstrates a remarkable filtration/backfiltration flux reaching values $>30$ $\mathrm{mL} / \mathrm{min}$ at a blood flow of $300 \mathrm{~mL} / \mathrm{min}$ and zero net filtration. Conclusion: The MCO dialyzer Theranova 400 appears to have a design optimized to perform expanded hemodialysis (HDx). karger@karger.com www.karger.com/bpu
(C) 2021 S. Karger AG, Basel 


\section{Introduction}

The evolution of hemodialysis technology has been stagnating for some years. Recently, the introduction of new medium cutoff (MCO) membranes has spurred new interest on potential improvements in medium- and long-term outcomes in patients undergoing chronic hemodialysis [1,2]. MCO membranes [3] present increased solute permeability with improvement of molecular weight retention onset (MWRO) while maintaining cutoff values adequate to limit possible albumin losses. MCO membranes are utilized in a dialysis technique defined "expanded hemodialysis" (HDx) that provides significant improvement in removal of large $(\mathrm{PM}>25$ and $<58 \mathrm{kDa})$ middle molecular weight solutes (LMM) responsible for symptoms and complications [4]. While solute clearances, sieving coefficients, and hydraulic permeability have been extensively studied, flow dynamic characteristics of hollow fiber hemodialyzers utilizing such membranes have not been studied in detail. This information may be required to correctly prescribe HDx and to optimize its operational parameters. Based on our experience of device evaluation in terms of performance, safety, and operational characteristics, we planned a thorough evaluation of the flow dynamic and cross-filtration characteristics of the new MCO hemodialyzer (Theranova 400; Baxter, Deerfield, IL, USA) aiming at gathering specific information useful for the correct and safe delivery of expanded hemodialysis with the MCO membrane.

\section{Materials and Methods}

The new technique called HDx (expanded hemodialysis) is made possible by the MCO membrane combined with a special hollow fiber geometry (reduced inner diameter) and a peculiar hemodialyzer design (length, cross-sectional area, and packing density). HDx is generally performed using the Theranova 400 (Baxter International Inc., Deerfield, IL, USA) filter unit. This is an MCO hemodialyzer designed specifically for HDx with a surface area of $1.7 \mathrm{~m}^{2}$. The membrane has an asymmetric 3-layer structure composed of polyarylethersulfone and polyvinylpyrrolidone blend, BPA free. It is characterized by uniform pore distribution and specific sieving properties: high MWRO and MW cutoff (MWCO) value lower than albumin [5]. MWRO is defined by the molecular weight value at which the sieving coefficient (in the absence of any gradient for diffusion) is 0.9 . MWCO is defined by the molecular weight value at which the sieving coefficient (in the absence of any gradient for diffusion) is 0.1 . The best performance of a membrane is achieved when the MWRO and the MWCO values are very close, which means a steep sieving curve and a sharp separation capacity. Because complex transport mechanisms are occurring inside the hollow fiber hemodialyzer during $\mathrm{HDx}$, it is important to define the flow dynamic conditions inside the blood compartment and the flow distribution inside the dialysate compartment. Besides defining the hydraulic permeability of the membrane and its sieving properties, a careful analysis of the segmental cross flow (direct filtration and backfiltration) along the length of the hollow fiber bundle is mandatory to understand how HDx operates. These were the objectives of the experimental setup created for the present study. Blood and dialysate flow distribution and internal transmembrane cross-filtration were studied with 2 separate imaging techniques: CT helical scanning and sequential and static scintigraphic imaging.

\section{Helical Scanning Technique for Blood and Dialysate Flow} Dynamic Analysis

Blood and dialysate flow distribution has been separately (before) and simultaneously (after) analyzed using a CT imaging technique with 2 different experimental setups. Circuits and filters were prefilled with heparinized blood $(\mathrm{Hct}=20 \%)$ and dialysate solution (Prismasol ${ }^{\circledR}$; Baxter International Inc., Deerfield, IL, USA). For each experiment, a batch of $200 \mathrm{~mL}$ of saline solution containing contrast medium (Visipaque $270 \mathrm{mg} / \mathrm{mL}$; GE Healthcare, Milano, Italy) diluted 1:4 was prepared. A dedicated syringe pump (Medrad Stellant CT Injection System; Bayer HealthCare, Whippany, NJ, USA) was used for injection in the circuit. Sequential imaging was obtained with a last-generation helical CT scanner (Revolution EVO 3.6; GE Medical System, Milwaukee, WI, USA) with a built-in software for static and dynamic evaluation of defined layers. The dialyzer was held in vertical position by a support inside the gantry in order to obtain a longitudinal section of the device. A 5-mm thick longitudinal layer of the dialyzer was analyzed with a specific software featuring $1 \mathrm{scan} / \mathrm{s}$. The scanning sequence started at the same time of the dye injection and lasted until the filter was completely filled. In the first experimental setup, the compartment under investigation was connected to an open circuit to have a single-pass configuration, and the other compartment was prefilled but closed (Fig. 1). Flow rates were $300 \mathrm{~mL} / \mathrm{min}$ for the blood compartment and $500 \mathrm{~mL} / \mathrm{min}$ for the dialysate compartment. Blood and dialysate compartments were analyzed in separate sessions. In the subsequent experimental setup, 2 peristaltic pumps connected to the circuit circulated simultaneously blood and dialysate in counter-current mode at 300 and $500 \mathrm{~mL} / \mathrm{min}$, respectively. The compartment receiving the dye solution was connected to a single-pass configuration circuit, and the other circulated in a closed loop to ensure that no net filtration could occur and zero fluid balance was maintained. The dialyzer was placed in the gantry in vertical position with blood running top-down and dialysate in bottom-up mode (counter-current configuration, Fig. 2). After reaching the steady state, a bolus of dye solution was injected in the selected compartment (prefilter immediately after the pump segment).

After acquisition of sequential images, quantitative analysis was carried out on the graphical reconstruction of the dye distribution pattern inside the studied compartment of the device. The central slice was selected and then analyzed over the duration of the acquisition. Regional distribution was assessed, and velocity was calculated as the ratio between 2 different regions of interest over the time required to reach the same relative increase in density (expressed in Hounsfield units). The analysis of the dye mass transfer zone (MTZ), that is, the distance from the maximal dye saturation to the absence of dye, was employed to describe the homogeneity of the flow distribution, for both compartments. The 


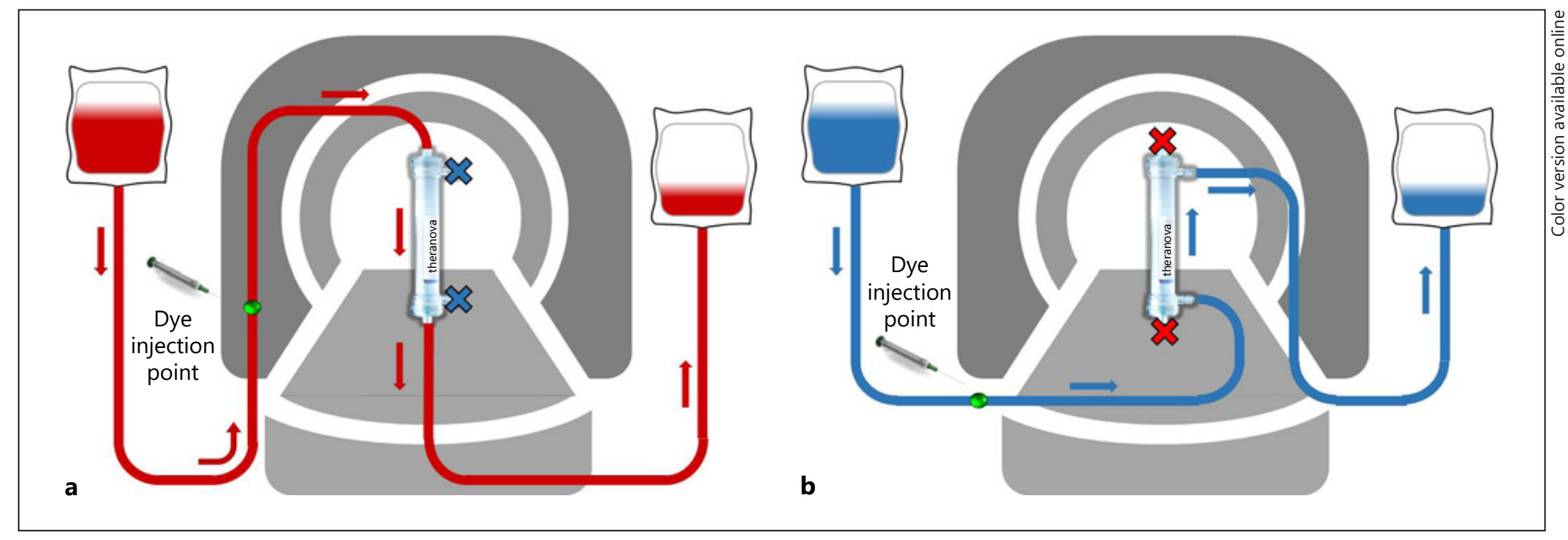

Fig. 1. Schematic representation of the first experimental setup. The dialyzer was held in vertical position and placed in the middle of the gantry. Blood and dialysate compartments were analyzed separately. a Dye solution was injected in the blood inlet line, and

flow was directed from the top to the bottom; the dialysate compartment was prefilled and sealed. b Dye solution was injected in the dialysate inlet line, and flow was directed from the bottom to the top; the blood compartment was prefilled and sealed.

Fig. 2. Schematic representation of the second experimental setup. The dialyzer was held in vertical position and placed in the middle of the gantry. Two peristaltic pumps flowed blood and dialysate in counter-current at 300 and $500 \mathrm{~mL} / \mathrm{min}$, respectively, and no net filtration. a Dye solution was injected in the blood inlet line, and flow was directed from the top to the bottom; the dialysate circulated in a closed loop. b Dye solution was injected in the dialysate inlet line, and flow was directed from the bottom to the top; blood circulated in a closed loop.

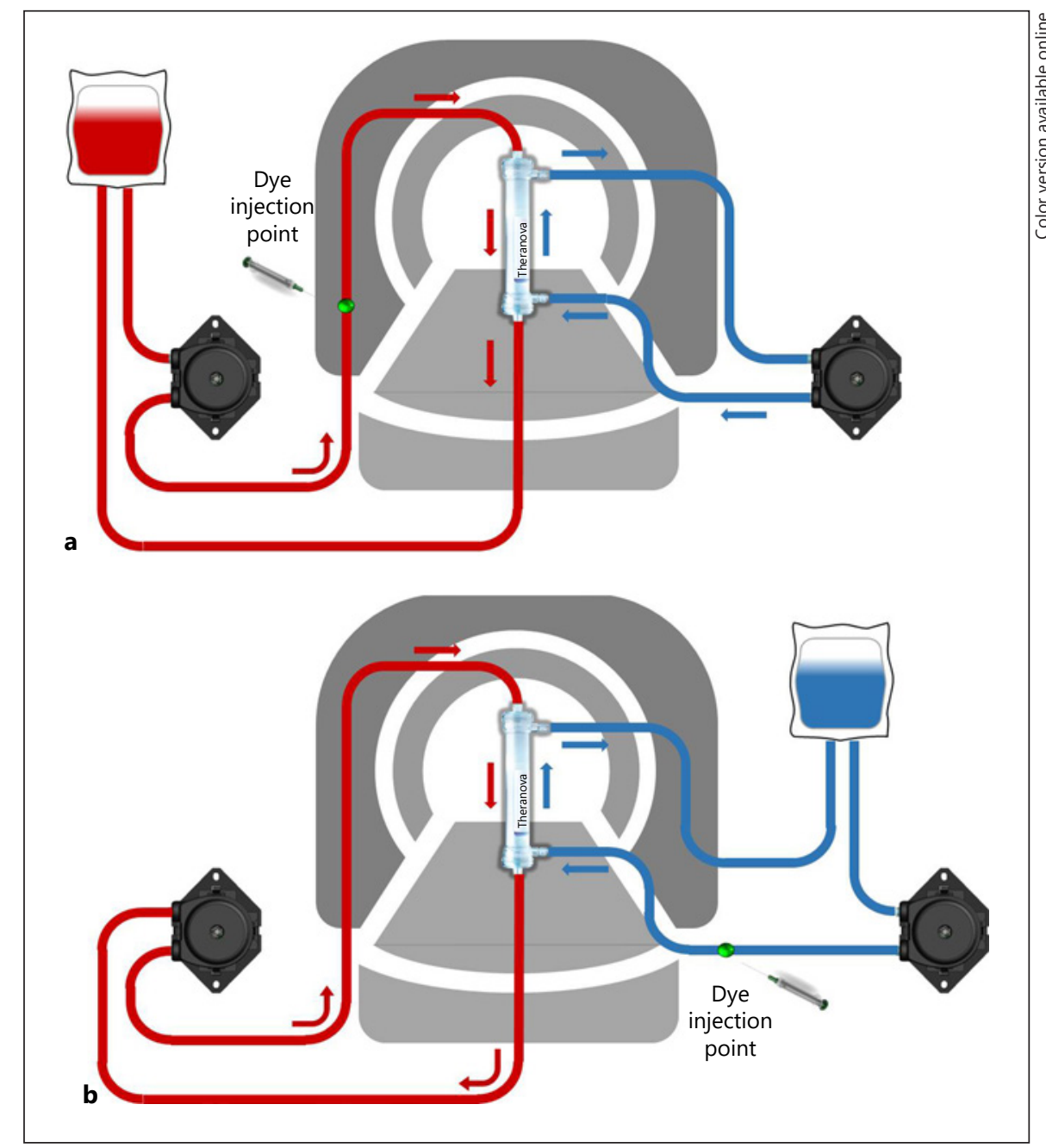




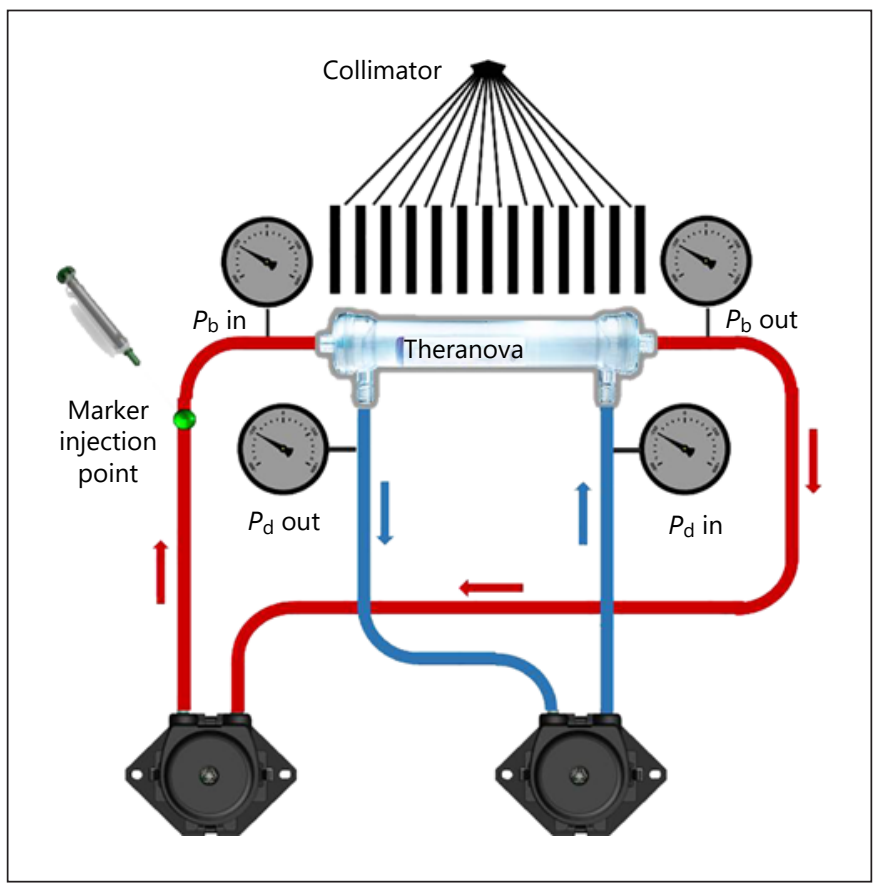

Fig. 3. The scintigraphic experimental setup. Dialyzer was laid on the gamma camera. Blood $(300 \mathrm{~mL} / \mathrm{min})$ and dialysate $(500 \mathrm{~mL} /$ $\mathrm{min}$ ) were circulated in a closed loop configuration ensuring zero net filtration. The tracer was injected in the blood line, upstream the inlet of the dialyzer. Pressures at inlet and outlet of the 2 compartments were monitored.

concept of the MTZ is derived from the studies carried out on sorbent cartridges where a dye solution is circulated through the sorbent bed. At any point of the cartridge, the MTZ is defined as the longitudinal distance between the cross-sectional point where a complete saturation of the sorbent occurs and the cross-sectional point where zero saturation is observed. Obviously, the longer the MTZ, the worse the quality of the unit design and its efficiency. The shorter the MTZ, the better the design of the unit and the utilization of the available sorbent bed. For a hemodialyzer, the MTZ can be calculated in the blood compartment by the longitudinal distance from the point where all fibers are perfused to the point where no fibers are perfused yet by the blood. In the dialysate compartment, the calculation is similar, but the dialysate flow is in the tortuous path external to the fibers. For this analysis, the injection of a bolus of dye into the blood and dialysate compartment is an excellent modality to evaluate the perfusion of specific areas of the dialyzer using sequential images achieved by a helical scanner.

\section{Sequential and Static Scintigraphic Study}

A different analysis was conducted employing a scintigraphic method to assess the internal filtration/backfiltration. As frequently stated in previous scientific studies, the distinction between diffusion and convection inside a hemodialyzer is practically impossible. The same is true for the evaluation of local cross flow at different points of the length of the hemodialyzer. Nevertheless, a unique method was established by our group in previous studies [6-8] to evaluate local fluxes of direct filtration and backfiltration.
The theory of this method is based on the assumption that changes in concentration of a nondiffusible marker molecule (proteinaceous) or a biological element (red cells) can only occur along the length of the hollow fibers as a consequence of a local change in the water content of the blood flowing inside the fibers. This can only be detected by a collimator of a gamma camera if the molecule or the biological element is labeled with a radioactive marker. In the present study, the dialyzer was placed on the horizontal support of the gamma camera and securely fixed to the collimator. Blood and dialysate were recirculated in a closed loop configuration ensuring zero net ultrafiltration (blood flow $300 \mathrm{~mL} / \mathrm{min}$ and dialysate flow $500 \mathrm{~mL} / \mathrm{min}$ ), as shown in Figure 3. A nondiffusible marker molecule (albumin macroaggregates labeled with 99Tc metastable, Macrotec; GE Healthcare, Milano, Italy) was injected in the blood compartment, and nuclear emission was recorded by using the gamma camera. Relative variations in concentration of the marker molecule along the length of the filter were used to calculate direction and amount of local cross-filtration at different points, as described in previous studies [6-8]. Images were acquired with a hybrid Discovery $670 \mathrm{NM} / \mathrm{CT}$ system manufactured by General Electric Medical System (Milwaukee, WI, USA).

As in the experiment described before [6], variations in marker molecule concentration were captured along the length of the hemodialyzers by using the gamma camera as counts. Since the marker remains confined in the blood compartment, changes in concentration reflect segmental variations of water content inside the fibers. Direct (IF) and reverse (BF) cross-filtration can therefore be estimated at each point of the dialyzer leading to cumulative calculation.

IF can be calculated by the following formula:

$$
\mathrm{IF}=\mathrm{Qb}(1-\mathrm{Hct}) \times\left(1-C_{\mathrm{in}} /\left(C_{\max }\right)\right)
$$

where $\mathrm{Qb}$ is the blood flow rate, Hct is the hematocrit, $C_{\text {in }}$ is the count of the marker molecule at the inlet of the dialyzer, and $C_{\max }$ is the peak count of the marker molecule along the length of the dialyzer.

$\mathrm{BF}$ can be calculated as follows:

$$
\mathrm{BF}=\mathrm{Qb}(1-\mathrm{Hct}) \times\left(1-C_{\text {out }} /\left(C_{\max }\right)\right)
$$

where $C_{\text {out }}$ is the count of the marker at the outlet of the hemodialyzer.

IF and BF are calculated using plasma flow since the marker molecule is confined to the plasma compartment. Hct and Qb were constant at the inlet and the outlet of the dialyzer confirming the experimental condition of zero net filtration. IF was equal to BF.

\section{Results}

Figure 4 displays the dye distribution pattern in blood and dialysate compartments during the first experimental setup: blue/green areas are free from dye solution, pink areas point out the presence of the dye solution, and $\mathrm{red} /$ yellow areas are transition zones. The digital imaging of the flow distribution pattern was obtained by computerized reconstruction of sequential images. In the blood compartment $(300 \mathrm{~mL} / \mathrm{min})$, flow distribution appears 

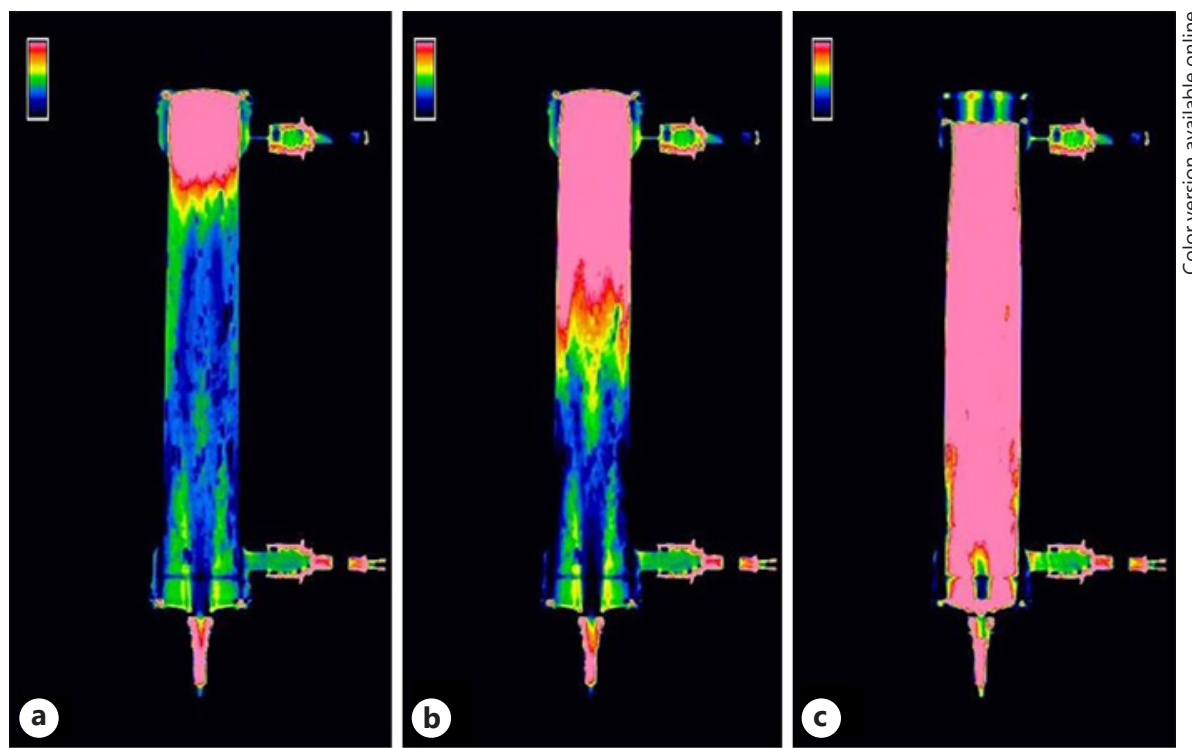

Fig. 4. Dye progression in blood $(\mathbf{a}, \mathbf{b}, \mathbf{c})$ and dialysate (d, e, f) compartments after 4 and $12 \mathrm{~s}$ and at total filling (33 $\mathrm{s}$ for blood and $28 \mathrm{~s}$ for dialysate), for the first experimental setup. Blue/green areas are free from dye solution, pink areas point out the presence of the dye solution, and red/yellow areas are transition zones.
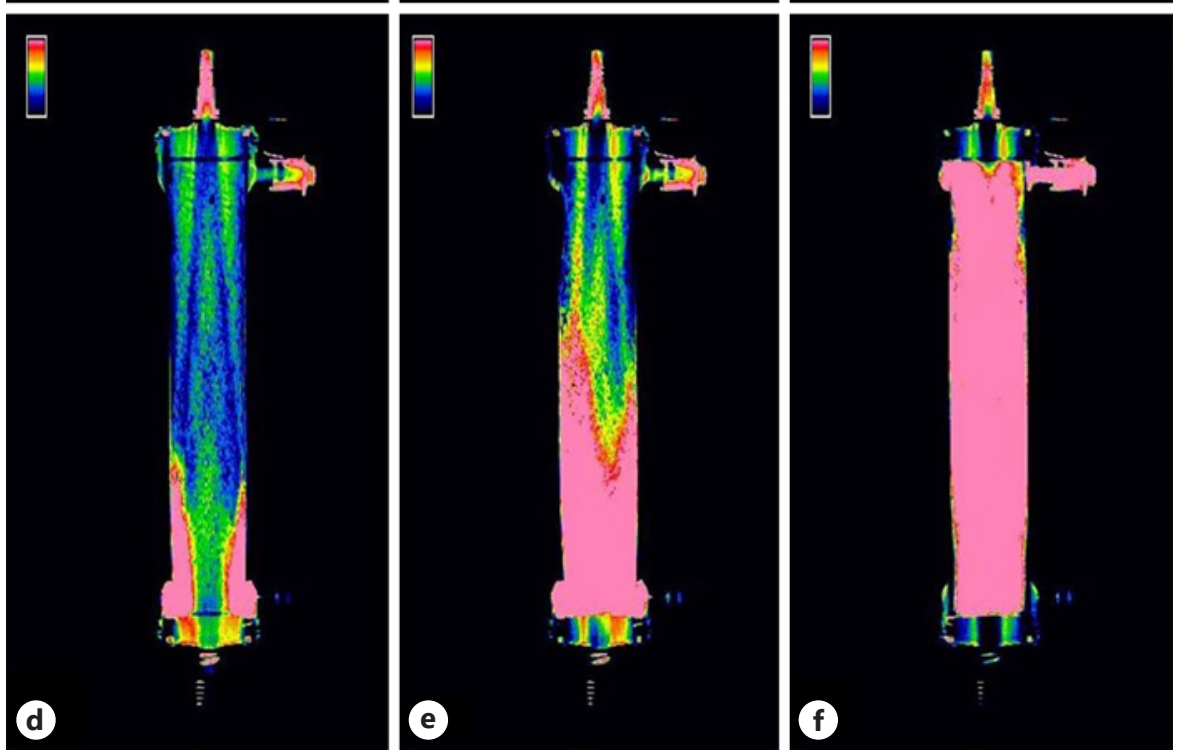

homogeneous, and complete filling of the fibers was reached after $33 \mathrm{~s}$. Minimal difference in velocity was observed between peripheral and central fibers. In the dialysate compartment $(500 \mathrm{~mL} / \mathrm{min})$, dye solution is distributed in the periphery first and then seeps in the fiber bundle and reaches the complete compartment filling after $28 \mathrm{~s}$. No dead space or irregularities are noticed from the images of the filled compartments, except for a black spot in the blood one, due to an air bubble artifact (Fig. 4c).

In the blood compartment, the velocity profile changes its shape along the length of the dialyzer. In the inlet blood port, the dye solution displays a homogeneous progression featuring a kind of plug flow configuration with an average velocity of $1.4 \mathrm{~cm} / \mathrm{s}$ (Fig. 4a). A small increase in differential velocity among the fibers was observed as the dye proceeds toward the outlet port. Peaks of velocity are displayed in the central $(1.1 \mathrm{~cm} / \mathrm{s})$ and in the extreme peripheral regions $(1.5 \mathrm{~cm} / \mathrm{s})$ of the dialyzer, while velocities as low as $0.6 \mathrm{~cm} / \mathrm{s}$ are observed in rare groups of intermediate fibers. The wall shear rates are 458,666 , and $276 \mathrm{~s}^{-1}$, respectively. These differences are absolutely acceptable and compatible with a well-designed blood compartment and a good flow distribution in the fiber bundle. Furthermore, the wall shear rate values are always above the limit where blood viscosity starts to increase significantly [9]. The very short length of the MTZ $(1.5 \mathrm{~cm})$ in the proximal part of the dialyzer (Fig. 5a) confirms the 


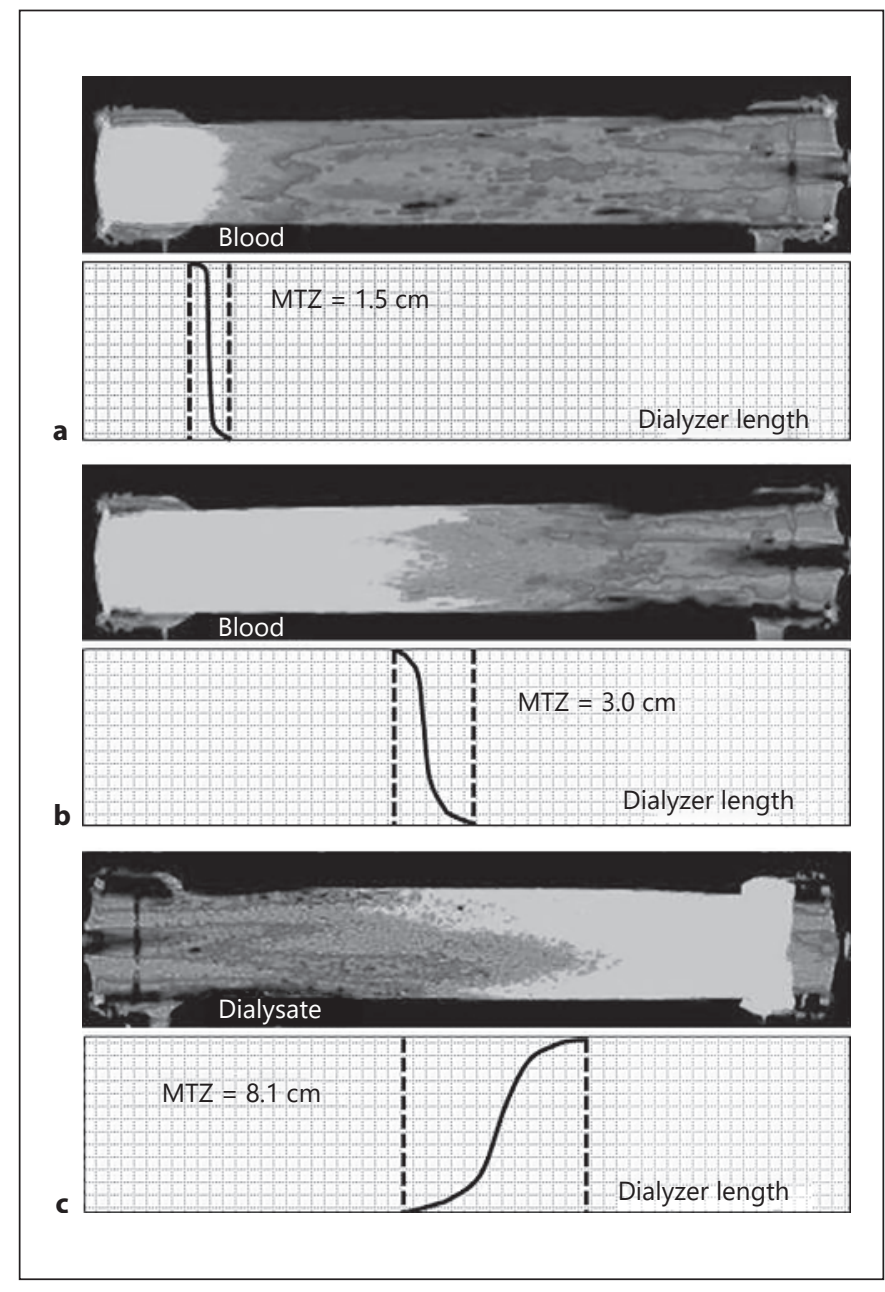

Fig. 5. MTZ in blood (a, b) and dialysate (c) compartments obtained from the first acquisition. MTZ represents the distance between the point of maximal dye saturation and the point of absolute absence of dye. MTZ, mass transfer zone.

plug flow; at half length of the dialyzer, MTZ is slightly longer but still within optimal values $(3 \mathrm{~cm})$ confirming the excellent utilization of all fibers of the bundle and a good distribution of flow with optimized flow velocity per single fiber (Fig. 5b).

In the dialysate compartment, dye solution flows initially along the case and through the outlying fibers with a velocity of $1.8 \mathrm{~cm} / \mathrm{s}$. When the solution begins to seep in the center of the fiber bundle, the velocity in the central region rises to $1.7 \mathrm{~cm} / \mathrm{s}$. Proceeding to the outlet port, the front of the velocity profile becomes more homogeneous with the optimal utilization of the whole cross-sectional area available for the flow (Fig. 4f). The dialysate MTZ presents a value of $8.1 \mathrm{~cm}$. While a moderate dispersion of local velocities is observed, the value is far below half
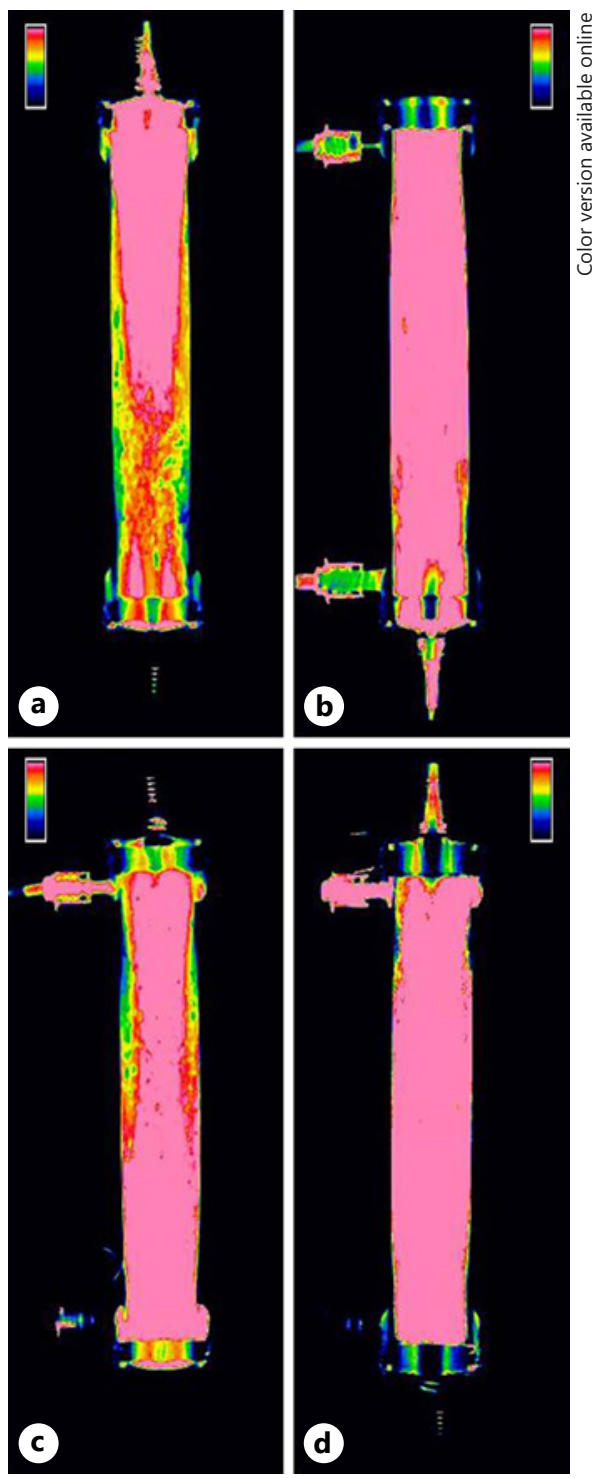

Fig. 6. On the left, CT images acquired after reaching the total filling in the blood (a) and dialysate (c) compartment in the countercurrent flow experiment. Visible differences are noticed if compared with the same images acquired in the first experiment $(\mathbf{b}, \mathbf{d})$. The reduction of dye intensity in the images on the left is the consequence of the internal/backfiltration phenomenon that occurs in counter-current flow configuration.

of the length of the dialyzer confirming optimal flow distribution of the dialysate (Fig. 5c).

Figures $6 \mathrm{a}$ and $\mathrm{c}$ report the images obtained from the second experimental setup, in which blood and dialysate circulated counter-current, simultaneously. Compared with the previous experiment (Fig. 6b, d), images display a perturbation of the dialyzer perfusion likely induced by the local transmembrane cross flow of dialysate and plas- 


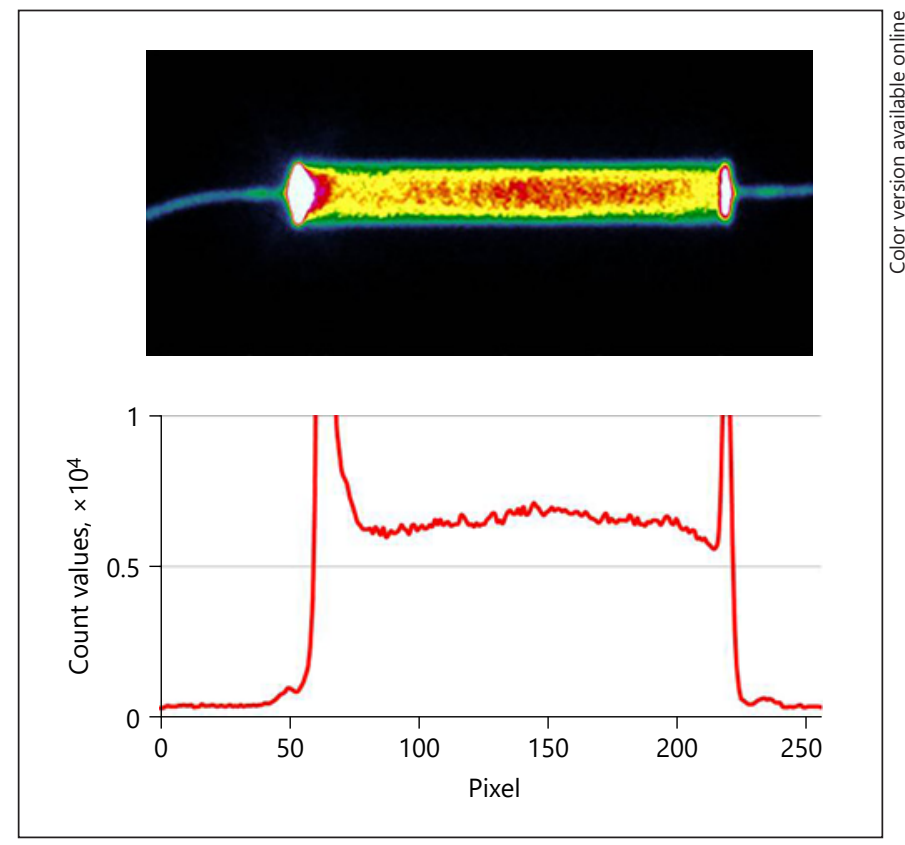

Fig. 7. Scintigraphic image of the dialyzer at the steady state and radioactivity count. The change in color corresponds to the relative variation in radiolabeled marker molecule activity in a numerical scale. Radioactive count is proportional to the marker concentration in blood. In the proximal part, the internal filtration causes the increase in marker counts while the backfiltration in the distal part brings back the radioactive counts to the inlet value, restoring the zero net filtration condition.

ma water. The blood flow distribution (blood flows from the top to the bottom) is homogeneous in the proximal half of the dialyzer while in the distal part, yellow areas describe the effect of backfiltration from the dialysate compartment into the hollow fibers. Dialysate compartment describes an analog effect (dialysate flows from bottom to top in counter-current with blood). Dye solution saturates the portion of the dialyzer close to the inlet (pink), while toward the middle of the dialyzer, the flow distribution pattern becomes slightly dishomogeneous due to ultrafiltration (plasma water cross flow from the blood into the dialysate compartment). This complementary behavior testifies the internal filtration and backfiltration phenomena. Considering the orientation of blood stream, in the proximal part of the dialyzer, plasma water crosses the membrane and the effect of the internal filtration causes a dilution of the dye solution in the dialysate compartment (Fig. 6c); in the distal part, instead, the reduction in dye intensity in the blood compartment is a consequence of backfiltration, the cross flow of the dialysate through the membrane into the fibers (Fig. 6a).
The kinetics of transmembrane cross flow are confirmed in the third experiment. Scintigraphic images (Fig. 7) display significant variation in radioactive count along the length of the dialyzer, due to local transmembrane cross flow of plasma water through the membrane in both directions. The increase in concentration of the marker molecule in the proximal part is ascribed to the direct filtration of plasma water from blood into the dialysate while the dilution in the distal part to backfiltration of the dialysate into blood. The turning point is observed at $53 \%$ of the dialyzer length. At steady state, the concentrations at inlet and outlet of the fiber bundle are identical, confirming the condition of zero net filtration. The calculation of relative changes of the marker molecule allows for the cumulative estimation of the amount of filtration and backfiltration inside the dialyzer under the selected experimental conditions.

The concentration of the marker molecule increases reaching a peak value $\left(C_{\max }\right)$ at half length of the filter and then decreases until the end of the fiber bundle reaching the same concentration observed at the inlet. This behavior demonstrates a proximal direct filtration (IF) and a distal backfiltration (BF). Calculated IF was $32.75 \mathrm{~mL} /$ min while BF was $32.46 \mathrm{~mL} / \mathrm{min}$. The slight difference is compatible with the error of the method. Filtration and backfiltration rates can increase or decrease depending on blood and dialysate flows and dialyzer surface area as reported in a previous publication.

\section{Discussion}

MCO membranes feature a characteristic sieving profile, with a high retention onset (MWRO corresponding to a sieving value of 0.9 for solutes up to a molecular weight of $11 \mathrm{kDa}$ ) value in a wide spectrum of molecular weights and a cutoff value proximal to the molecular weight of albumin. This innovative profile guarantees an enhanced clearance of medium-large molecular weight solutes compared to standard high-flux dialyzers and negligible losses of albumin compared to the high cutoff membrane. MCO membranes are commonly applied in a hemodialysis modality defined HDx where a significant convective exchange is obtained inside the dialyzer by an enhanced mechanism of internal filtration/backfiltration. In the case of the Theranova dialyzer, this phenomenon is optimized thanks to the combination of the sieving properties of the membrane, its hydraulic permeability, and the peculiar geometry of the fibers with the reduced inner diameter [10]. In fact, not only the reduced
Lorenzin/Golino/de Cal/Pajarin/ Savastano/Lupi/Sandini/Fiorin/Ronco 
inner diameter of the fibers causes an increased pressure drop in the blood compartment maximizing the positive transmembrane pressure in the proximal part and the negative transmembrane pressure in the distal part of the dialyzer but also guarantees an excellent average flow velocity and wall shear rate inside the fibers. While these mechanisms were hypothesized and theoretically estimated, they were not fully demonstrated so far. Indeed, the condition for an optimized filtration/backfiltration kinetics is the homogeneous distribution of blood and dialysate flows in the relevant compartments.

The homogeneous pattern of blood distribution at the inlet port and along the fiber bundle represents a proof of an optimal design of the distributor and the entire blood compartment of the dialyzer. This ensures a good performance of the filter in the diffusion mode and guarantees the optimal utilization of the available surface area. Adequate flow distribution and high wall shear rates contribute to minimize the formation of a protein layer at the blood membrane interface. The occurrence of a significant concentration polarization phenomenon with consequent reduction of membrane permeability is mitigated by the utilization of the dialyzer in the hemodialysis mode (HDx). In fact, excessive convective rates such as those achieved in the hemodiafiltration mode would contribute to impair membrane permeability by the formation of a protein cake onto the internal surface of the fibers. This phenomenon leads to the formation of a new contact surface whose thickness is added to that of the original membrane and interferes with the final permeability of the membrane in vivo. This unwanted effect is prevented by high wall shear rates in all fibers which contribute to reduce the thickness of the protein boundary layer and improve membrane hydraulic and sieving permeability. Diffusion is described by Fick's law and is inversely related to the distance of the 2 compartments. Higher shear rates contribute to maintain the diffusion distance between blood and dialysate within minimal values. In convection, the shear rates contribute to keeping the polarization layer very thin. At $300 \mathrm{~mL} / \mathrm{min}$ of blood flow, Theranova 400 showed shear rates of $458 \mathrm{~s}^{-1}$ in the central region, $276 \mathrm{~s}^{-1}$ in the middle regions, and $666 \mathrm{~s}^{-1}$ in the periphery; even the lowest value results $>200 \mathrm{~s}^{-1}$, threshold for a Newtonian behavior, ensuring optimal performances.

The distribution of the dialysate flow outside the fibers is also optimized as demonstrated by the dynamic images where the dye appears to be homogeneously distributed in the entire cross-sectional area of the dialysate compartment. Thus, any blood-to-dialysate flow mismatch is avoided, and the surface area available for the exchange is maximized. These observations are of remarkable importance also in light of the cross flow kinetic results obtained with the nuclear scintigraphic method. Our results confirm a significant amount of filtration and backfiltration inside the dialyzer at zero net filtration $>30 \mathrm{~mL} / \mathrm{min}$. This means that in presence of higher blood flows, higher surface areas, and higher net filtration rates $(15-20 \mathrm{~mL} / \mathrm{min})$, direct fluid cross flow may reach values around $50 \mathrm{~mL} /$ $\mathrm{min}$. This is achieved in presence of a wall shear rate in all fibers sufficiently high to maintain blood viscosity at minimal levels (Newtonian behavior) and a significant cleaning effect at the blood membrane interface with negligible concentration polarization of plasma proteins. Such effect results in a maintenance of the sieving properties of the membranes throughout the dialysis session. Internal filtration between 30 and $50 \mathrm{~mL} / \mathrm{min}$ combined with the sieving characteristics of MCO membranes allows for convective clearance values of medium-large molecules equal or even superior to those achieved in high-volume online hemodiafiltration, without need of fluid replacement and very high filtration fractions inside the hemodialyzer. In conclusion, this study makes the basis for the utilization of MCO membranes in expanded hemodialysis maximizing the benefits of internal filtration while maintaining the simplicity and safety [11] of high-flux dialysis configuration and blood flows in the range of $300 \mathrm{~mL} / \mathrm{min}$.

\section{Acknowledgment}

The authors are grateful to the International Renal Research Institute of Vicenza.

\section{Statement of Ethics}

The study is exempt from ethical committee approval since the whole experiment was carried out in vitro, and it does not involve human beings or animals and not contain medical information of patients.

\section{Conflict of Interest Statement}

Claudio Ronco has received consultancy and speaking fees from ASAHI, Astute, bioMerieux, B. Braun, Baxter, FMC, Toray, GE, OCD, Jafron, and Medtronic. All other authors have no conflicts of interest to declare.

\section{Funding Sources}

No funding sources to declare. 


\section{Author Contributions}

C. Ronco, A. Lorenzin, A. Lupi, and S. Savastano contributed in the study design and M. de Cal, A. Sandini, and F. Fiorin in experimental settings and sample measurements; A. Lorenzin, G. Golino, and G. Pajarin carried out the experiments; A. Lorenzin analyzed the data; A. Lorenzin and C. Ronco contributed in the interpretation and the writing process. All authors approved the final version of the manuscript. Each author contributed important intellectual content during manuscript drafting or revision and accept accountability for the overall work by ensuring that questions pertaining to the accuracy or integrity of any portion of the work are appropriately investigated and resolved.

\section{References}

1 Boschetti-de-Fierro A, Voigt M, Storr M, Krause B. Extended characterization of a new class of membranes for blood purification: the high cut-off membranes. Int J Artif Organs. 2013;36:455-63.

2 Ronco C, Clark WR. Haemodialysis membranes. Nat Rev Nephrol. 2018;14(6):394410.

3 Rosner MH, Reis T, Husain-Syed F, Vanholder R, Hutchison C, Stenvinkel P, et al. A new definition and classification of middle molecules and their role in uremia: an expert consensus conference. CJASN. Forthcoming. 2021.

4 Wolley M, Jardine M, Hutchison CA. Exploring the clinical relevance of providing increased removal of large middle molecules. Clin J Am Soc Nephrol. 2018;13:805-14.
5 Cozzolino M, Ronco C. Medium cut-off membranes: incremental or quantum leap innovation in haemodialysis? Blood Purif. 2020; $1-4$.

6 Ronco C, Brendolan A, Feriani M, Milan M, Conz P, Lupi A, et al. A new scintigraphic method to characterize ultrafiltration in hollow fiber dialyzers. Kidney Int. 1992 May; 41(5):1383-93.

7 Fiore GB, Guadagni G, Lupi A, Ricci Z, Ronco C. A new semiempirical mathematical model for prediction of internal filtration in hollow fiber hemodialyzers. Blood Purif. 2006;24(56):555-68.

8 Lorenzin A, Neri M, Lupi A, Todesco M, Santimaria M, Alghisi A, et al. Quantification of internal filtration in hollow fiber hemodialyzers with medium cut-off membrane. Blood Purif. 2018;46(3):196-204.
9 Ronco C, Levin N, Brendolan A, Nalesso F, Cruz D, Ocampo C, et al. Flow distribution analysis by helical scanning in polysulfone hemodialyzers: effects of fiber structure and design on flow patterns and solute clearances. Hemodial Int. 2006 Oct;10(4):380-8.

10 Ronco C, Brendolan A, Lupi A, Metry G, Levin NW. Effects of a reduced inner diameter of hollow fibers in hemodialyzers. Kidney Int. 2000;58:809-17.

11 Weiner DE, Falzon L, Skoufos L, Bernardo A, Beck W, Xiao M, et al. Efficacy and safety of expanded hemodialysis with the theranova 400 dialyzer: a randomized controlled trial. Clin J Am Soc Nephrol. 2020;15(9):1310-9. 